\title{
Improving children"s attention with focus training and meditation
}

The widespread use of increasingly sophisticated electronic devices and the accessible through these devices has been linked to decline in people's ability to pay attention to a single task or stimulus for prolonged periods of time. This might particularly true for children who grew up in the digital era, as a growing number of these in the struggle to concentrate mind, Kang-Ming Chang Yi-Jung Lai and colleagues at Asia University, Taiwan have recently investigated the potential of focus training strategies and meditation for improving the attention of elementary school students.
$T_{\text {oday, people have access to }}^{\text {an unprecedented amount }}$. of information through their smartphones, tablets, portable While digital technologies allow people to instantly learn about different topics and communicate with others around the world, their widespread use has considerably reduced the appeal of experiential and in-person learning.

Over the past decade or so, many educators have observed a decline in the performance and concentration of children in both elementary and secondary school. This decline has often been linked to a reduction in attention, which could in turn of digital technology. Many resead have thus been conducting studies aimed ave thus been conducting studes als attention and its impact on children's

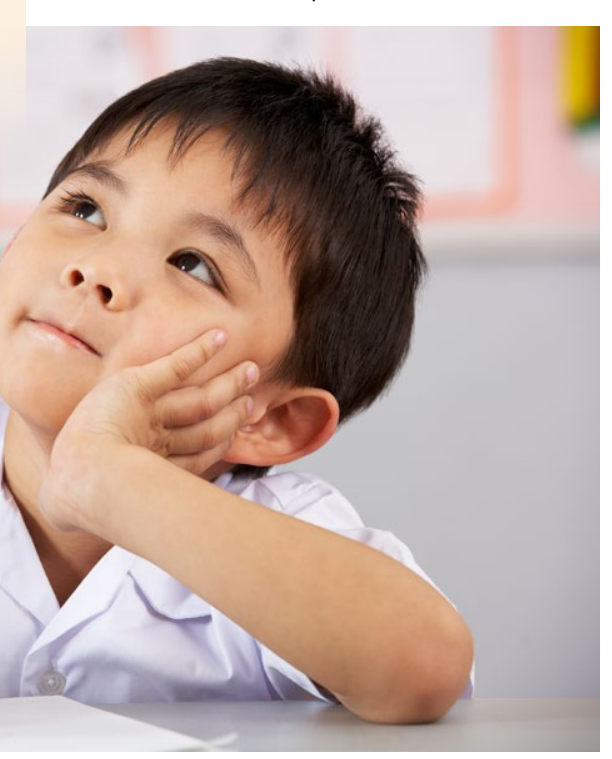

academic performance, while also trying identify strategies that could improve attention over time.

Drs Kang-Ming Chang and Yi-Jung Lai, in collaboration with colleagues at Asia University and at other institutes in Taiwan, have been trying to identify practices that could improve the attention of elementary school students. In one of their most recent studies, they specifically .

\section{UNDERSTANDING AND}

\section{MEASURING ATTENTION}

introduced by Sol of Attention, in 1987, divides attention into five distinct dimensions focused atention, sustained attention, selective attention, alternating attention, and divided attention. The term focused attention refers to a person's

Sustained attention is what allows people to consistently respond to stimuli during a prolonged or repetitive activity. The term selective attention, on the other hand, refers to a person's ability to direct his/ her behaviour or cognitive efforts towards a specific task or stimulus, even in the presence of distractions, whether externa e.g., sounds, movements, environmenta changes) or internal (e.g., worries, thoughts, etc.). Alternating attention is the

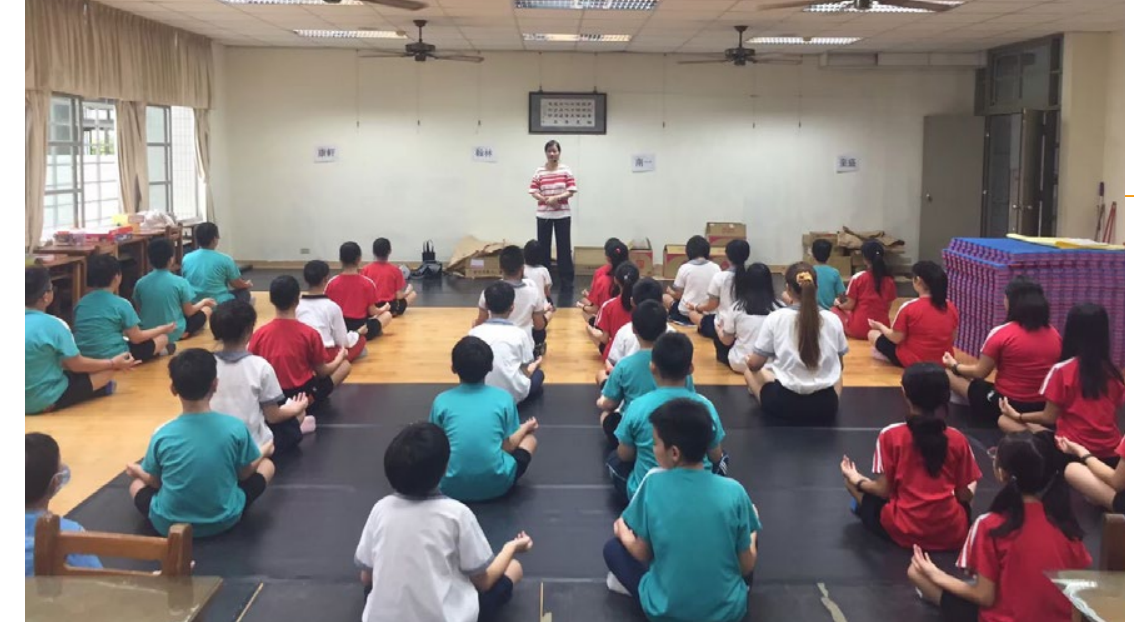

Drs Chang and Lai investigated the effects of focus training and meditation on

that require different cognitive abilities. Finally, divided attention is what allows people to simultaneously focus on

multiple tasks.

Over the years, researchers have devised different instruments and questionnaires that can be used to assess these differen Chang, taind attention of participants using a variety attrons, ranging from attention scale questionnaires to wearable devices and eye-tracking tools.

FOCUS TRAINING TO IMPROVE THE ATTENTION OF ELEMENTARY SCHOOL CHILDREN

In their recent study, Chang and Lai evaluated the effectiveness of a strategy for increasing people's attention that they refer to as fixation focus training

by specifically using it to improve the attention of elementary school students. To do this,
they asked groups they asked groups il and 12 year their attention on a

specific point on the wall in front of the for approximately 20 minutes, as they followed specific instructions.

Essentially, the children were asked to perform certain physical movements while paying particular attention to different parts of their body and simultaneously concentrating on the same point on the wall. For instance, they were asked to throw both of their hands backwards while standing on their toes and slightly tilting their hea backwards, all this as they focused The resentors on the hace. perform a seris of these moventont while focusing their attention on different areas of the body.

Once this dynamic part of the training was complete, the children sat down in a crosslegged position for 10-15 minutes, with their hands gently placed on the knees, their back straight and their eyes gently as 'static focus training' In other words as 'statc focus traing'. In thir words, on specific parts of their body (eg, the heart, stomach, throat, etc) while sitting still. Towards the end of this meditative practice, the researchers offered children the choice to focus on any body parts of their choice. Finally, all participants took part in a discussion session where they shared their experiences with one another.

The children attended one session per week for 12 weeks. The researchers

Chang and Lai examined the effects of focus training and meditation

on children's ability to concentrate for long periods of time.

assessed their attention before and after the training, using the Attention questionnaire-based approach to measure the five dimensions of attention outlined by Sohlberg and Mateer. They also

政 had attended the focus training sessions in the sessions.

THE BENEFITS OF FIXATION TOCUS TRAINING FOR CHILDREN'S ATENT AND WELLBEING attention of children who attended fixation focus training on a regular basis appeared to improve over time, particularly their scile the scores of children in the experimental and similar before the training, the attention group improved significantly after the focus training.

More specifically, the researchers found that the total attention mean DIFF value (defined as the post-test results minus the pretest results) of children in the experimental group was 27.20 , which is significantly $(p<0.05)$ higher than the 18.22 mean DIFF value observed in the co. $<05$ ) than in the control experimental group respectively) and so was ( 18.07 and 5.24 ttention mi an values of 2038 and 832 , respectively).

In other words, after the training children specific stimuli in their surroundings and they were better at focusing their attention on a specific stimulus even in the presence of external distractions (e.g., noises) or

Moreover, $54.1 \%$ of the children corted improvements in their

chestron during school lessons, $29.1 \%$ an enhanced ability to fall asleep at night, $8.4 \%$ said they felt more relaxed, $4.2 \%$ said
their stress had reduced and $4.2 \%$ said their chest pain focus training protocol used by Chang and Lai parly resembles mindfulness meditation techniques, which were found to have numerous beneficial effects and are employed in numerous therapeutic settings.

The results gathered by Chang and Lai highlights the potential of the focus training they developed. However, as they evaluated the attention of students using a questionnare primanly used in Taiwan, they hope to ultimately repeat their study utilising diferent instrant ins and questionnaires to assess changesin attention, as this would improve their validity. 


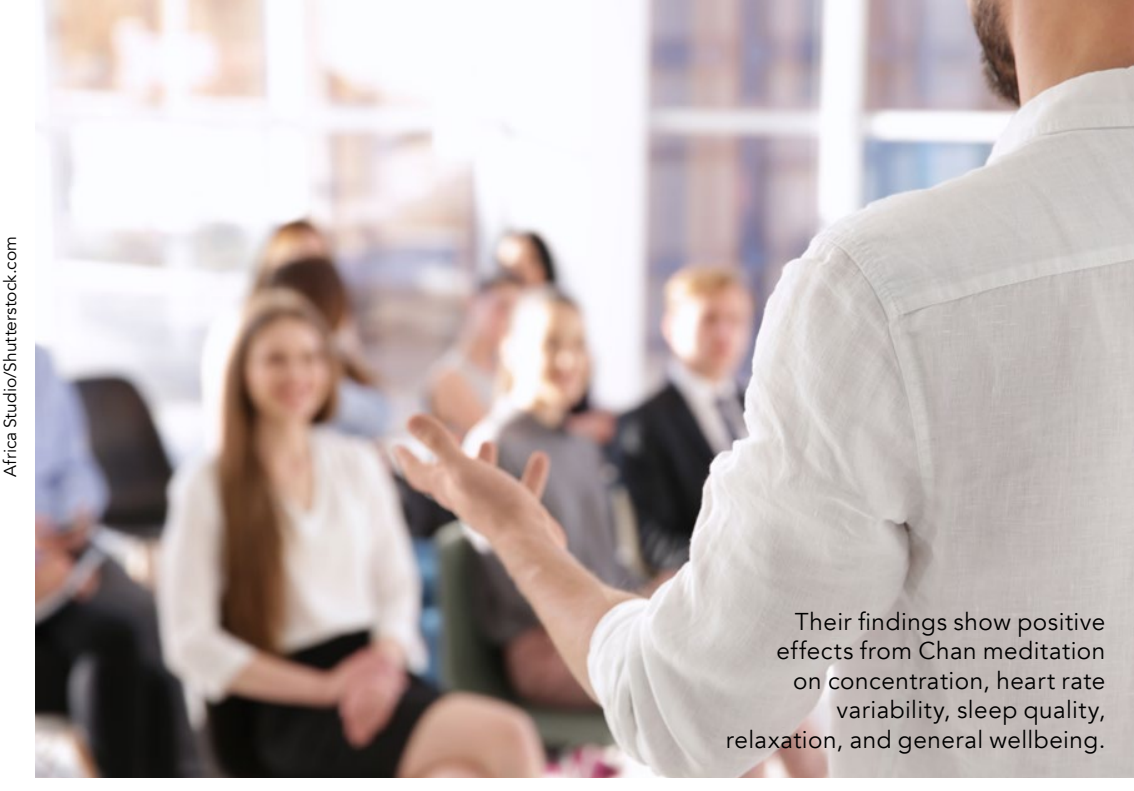

ADDITIONAL RESEARCH INVESTIGATING THE EFFECTS OF MEDITATION ON ADULTS In addition to exploring the effects of focus training and meditation on children's attention, Drs Chang and Lai effects of meditation practice on adults.

For instance, they organised two face-to-face and video-based Heart Chan meditation courses for both experienced and inexperienced adutt meditators, led by a Chan master. Chan is a Chinese school of Mahâyāna Buddhism and the Heart practice specifically asks meditators to focus their attention on Chakra points in the body. Interestingly, they found that both the heart rate and biological heart rate variability age of participants decrease significantly after they completed the meditation courses, regardless of whether they attended sessions in person or via video and even if they had no prior experience with meditation. In another study, Chang and Lai used ability of 306 ad ult participants to focus their gaze on a specific point in space

(at the centre of three concentric circles) for one minute. Interestingly, they found that those who could focus their gaze better had lower systolic blood pressure and reported sleeping beter at night. As gaze concentration is a crucial aspect of heart Chan medition, hese fits fixation focus training.

The researchers also conducted a study aimed at evaluating the physical stillness of adults as they practiced Cha Ding meditation, which asks people to focus on the ten energy points or

experienced meditators were able to emain still for longer periods of time.

THE POTENTIAL OF FOCUS TRAINING AND MEDITATION ATTENTION IN THE DIGITAL Overall, the results gathered by CRA Lai and their colleagues over the past few years highlight the possible effects of consistent Chan meditation on concentration, heart rate variability, sleep quality, relaxation, and general wellbeing.

Their most recent study specifically explored the impact of weekly meditation on the concentration of elementary school children. Their findings suggest that a combination of meditation and dynamic attention training could greatly benefit children, as it could help them to tast, lessonn to focus thim periods of time.

In the future, these results could pave the way for further studies aimed at investigating the effects of meditation practices on children's concentration. and academic performance. In addition,

These results could pave the way for further studies aimed at investigating the effects of meditation practices on children's concentration and academic performance.

centres outlined by the Chen school Physical stillness was as 'mailuns'. accelerometers that participants wore on their arms and chest Interestingly, the researchers observed that more

the work of Chang and Lai could inspire more schools and educators worldwide and meditation sessions, as a means to improve the attention of students and enhance their learning over time.



\section{Behind the Research} 悬

Dr Kang-Ming Chang

E: changkm@asia.edu.tw

Research Objectives

Dr Kang-Ming Chang's research is devoted to biomedical signal processing and Al. His recent interests include attention DrYi-Jung Lai's research is concerned with children's health and learning through focus and meditation training.

\section{Detail}

Dr Kang-Ming Chang is Associate Professor at the Department of Computer Science and Information Engineering, Asoon

Dr Yi-Jung Lai is Associate Professor at the Department of

\section{References}

Chang, K., Chueh, M., Lai, Y. (2020). Meditation Practice Improves Short-Tem, Changes in Hear Rate Variability. International Journal of Environmental Research and Public Health, 17, 2128. doi:10.3390/jijerph17062128

Lai, Y., Chang, K. (2020). Improvement of Attention in Elementary School Students through Fixation Focus Training (S) Environmental Research and Public Health, 17, 4780. doi:10.3390/ijerph17134780

Chang, K., Chueh, M. (2019). Using Eye Tracking to Assess Gaze Concentration in Meditation. Sensors, 19, 1612 doi:10.3390/s19071612

Chang, K., Chun, Y., Chen, S., Lu, L., Su, H., Liang, H., Santhosh, J., Ching, C., Liu, S. (2016). The Evaluation of Physical Stillness with Wearable Chest and Arm Accelerometer during Chan Ding Practice. Sensors, 16, 1126. doi:10.3390/s16071126

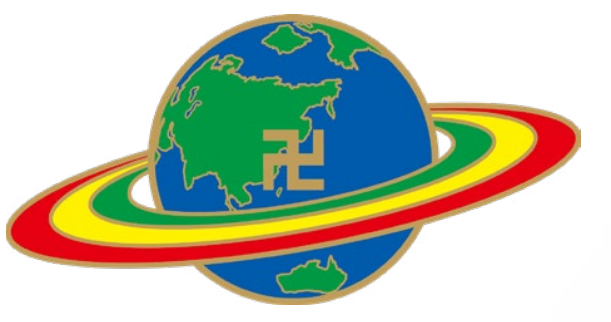

Early Childhood Educare, Wu Feng University.

Collaborators

Special thanks to the help of Shakyamuni Buddhist Foundation Chueh Miao-Tien, the 85th Patriarch of the Order of Chan since

\section{Personal Response}

\section{Based on the findings gathered so far, what training} strategies and meditation practices can help to improve children's attention the most, and how do

II Original attention training is based on Shakyamuni huddhist Foundation in Taiws:

People in United States can visit Heart Chan, a spiritua organisation based in the United States that shares the

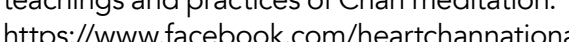

If anyone is interested about the practical teaching of focus attention training for elementary school children,
please contact DrYi-Jung Lai, yrlai@wfu.edu.tw

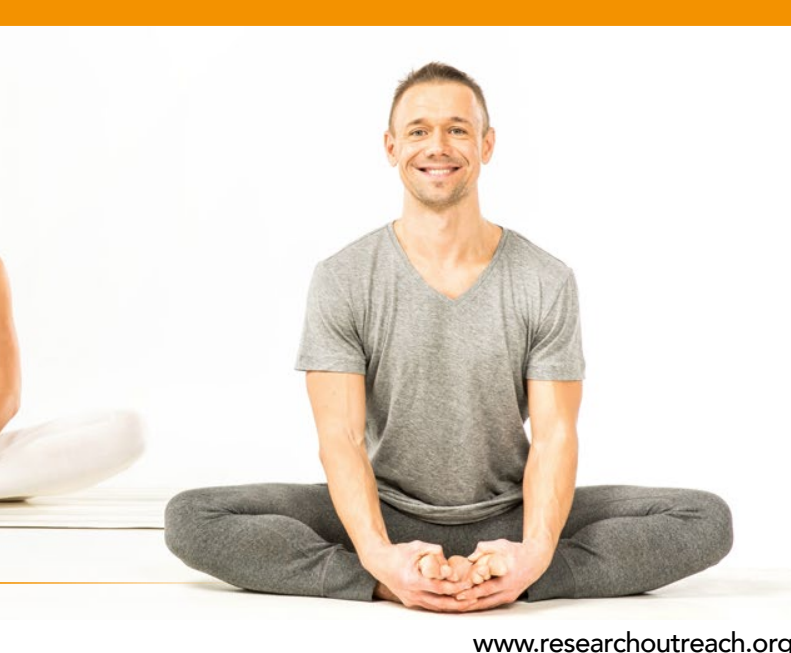

